\title{
Agent-based models and individualism: is the world agent-based?
}

\author{
David O'Sullivan and Mordechai Haklay \\ \{david.osullivan,m.haklay\}@ucl.ac.uk \\ Centre for Advanced Spatial Analysis, University College London \\ 1-19 Torrington Place, London WC1E 6BT, United Kingdom
}

\begin{abstract}
Agent-based models (ABM) are an increasingly popular tool in the social sciences. This trend seems likely to continue, so that they will become widely used in geography and urban and regional planning. We present an overview of examples of these models in the life sciences, economics, planning, sociology, and archaeology. We conclude that agent-based models strongly tend towards an individualist view of the social world. This point is reinforced by closer consideration of particular examples. This discussion pays attention to the inadequacy of an individualist model of society with reference to debates in social theory. We argue that because models are closed representations of an open world it is important that institutions and other social structures be explicitly included, or that their omission be explained. A tentative explanation for the bias of agent-based models is offered, based on an examination of early research in Artificial Intelligence (AI) and Distributed Artificial Intelligence (DAI) from which disciplines the approach is derived. Some implications of these findings are discussed. We indicate some useful research directions which are beginning to tackle the individualism issue directly. We further note that agent-based models' underlying assumptions are often hidden in the implementation details. We conclude that such models must be subject to critical examination of their assumptions, and that model builders should engage with social theory if the approach is to realise its full potential.
\end{abstract}
"BRIAN: $\quad$ Look. You've got it all wrong. You don't need to follow me. You don't need to follow anybody! You've got to think for yourselves. You're all individuals!

FOLLOWERS: Yes, we're all individuals!

BRIAN: $\quad$ You're all different!

FOLLOWERS: Yes, we are all different!

DENNIS: I'm not."

Monty Python's Life of Brian

\section{Introduction}

Agent-based models are a relatively new arrival among the methods available to geographers and urban planners hoping to extend our understanding of societies in different spatial contexts. They are one of the new computational techniques (others include cellular automata and genetic algorithms) being pioneered in the 'complexity sciences', which increasingly dominate both academic and 'popular' science bookshelves. In this paper we introduce agent-based models and modelling to the wider research community in the spatial sciences, at the same time raising some important interpretative 
and philosophical issues concerning social theories underlying these models. Although in places we are highly critical of agent-based models in the social sciences, we are enthusiastic about the possibilities of such models, while remaining wary of some of the hidden assumptions they frequently embody. Indeed, the impetus to write this paper arose from our own experience with building an agent-based model - the STREETS model of pedestrian behaviour in complex spatial environments (Schelhorn et al., 1999). Concerns, ideas and question raised by that process, have been the starting point for our enquiry into the biases and origins of agent-based models.

A precise definition of agent-based models is elusive, but obviously hinges on the meaning we attribute to the term 'agent'. Rather than review various proposed definitions', we direct the reader's attention to the many examples discussed below, and for now restrict ourselves to a workable outline definition. An agent can be thought of as an autonomous, goal-directed software entity. Agents' autonomy is constrained by the fact of their construction by human programmers but in this context means that they pursue their goals in an open-ended manner. A multi-agent system - the context in which the agent concept has been developed - is usually considered to be a collection of "self-contained problem solving systems capable of autonomous reactive, pro-active, social behaviour" (Lomuscio, 1999). Multi-agent systems (MAS) are not models but problem solving systems. In many MAS, agents incorporate sophisticated artificial intelligence techniques whereby they learn new ways to attain their goals. The most generic application for software agents is information retrieval for some human client. Thus agent software might take on the laborious task of trawling the internet for information likely to be of interest to its human client (Rodrigues and Raper, 1998 have examined this application in a spatial context). In pursuing this goal it may learn its client's preferences, and also encounter other agents. Communication with other agents is likely to significantly enhance its ability to find what it is looking for. Thus communication capability is programmed into agents - and in some accounts is a required feature.

An agent-based model or simulation removes agents from this 'real-world' context, and places them in an artificial world. Typically an agent-based model might have several hundred agents interacting in an artificial world representing some real world location or social institution (a market, for example). The agent modeller programs agents with plausible rules governing their behaviour and examines model outcomes to obtain insight into the real world situation modelled.

It seems clear that geographers and planners are well-placed to investigate such models in both theoretical and substantive ways, contributing to the development of the spatial aspects of these models, and examining the assumptions and theories which underlie them. It is in this spirit that this paper is presented. In section 2 we overview the rapidly expanding field of agent-based models taking in examples from biology, urban and regional planning, economics, sociology, and archaeology. Here the ontological and theoretical commitments of agent-based models begin to emerge. In section 3 we build on these insights by examining more closely the implicit philosophical commitments of the approach. We concentrate on one issue in particular: a distinct bias towards individualist perspectives. This is associated with a focus on one-way emergence of social phenomena from the aggregate activity of individuals. In section 4 we propose a tentative explanation for these biases based on a closer examination of the technological origins of agent-based models, and on influential intellectual positions regarding contemporary social developments. In section 5 we examine the implications of these findings for the 
construction of agent-based models in the social sciences, and direct attention to some recent perspectives and approaches which we feel are useful. In section 6 we draw some final conclusions.

\section{Overview of agent-based modelling}

Alongside much else with its roots in the development of information technologies, agentbased modelling is a rapidly developing field, already well beyond the scope of any limited survey, especially in light of its appearance in the literature of a number of different disciplines. A good sense of the state of these efforts is obtained from various edited volumes (see for example Gilbert and Conte, 1995, Gilbert and Doran, 1994, Conte et al., 1997, Sichman et al., 1998), and review articles (Tesfatsion, 1997). Gilbert and Troitzsch (1999) provide a good introduction in the wider context of social simulation. The definitive example of agent-based modelling technology is provided by the Santa $\mathrm{Fe}$ Institute's Swarm simulation toolkit which is available online (Minar et al., 1996). Also gaining in popularity is the StarLogo software developed at the MIT Media Lab (Epistemology and Learning Group, 1999, Resnick, 1994). Here we describe some examples at a level of detail intended to give an impression of the scope of the approach. Other examples are to be found by consulting the literature cited.

\subsection{The flocking behaviour of animals}

One of the earliest examples of applying an agent-based approach to a previously difficult problem is Reynolds's (1987) Boids model. This attempts to simulate the flocking behaviour of birds (the name Boid is a contraction of bird and android), and other 'flocking' animals. Reynolds's computer program has agents (boids) moving in a space following three 'steering rules': (i) avoid crowding nearby boids, (ii) steer towards the average heading of nearby boids, and (iii) steer towards the average position of nearby boids. Reynolds's aim was to produce a "believable animation of simulated flocks", and gives some sense of how the approach works. However, he also suggests that

“[i]t might be possible ... to perform controlled and repeatable experiments with 'simulated natural flocks'. A theory of flock organisation [could] be unambiguously tested by implementing a distributed behavioural model and simply comparing the aggregate motion of the simulated flock with the natural one."

(Reynolds' 1987, page 32)

Other more directly biologically motivated agent models are described in Resnick (1994) and Levy (1992). More recently, similar models have been integrated into geographic information systems (GIS) to assist in the management of herds of endangered species (Westervelt and Hopkins, 1999).

\subsection{Human movement patterns}

A similar set of ideas to those of the Boids model has been applied to human pedestrian behaviour, particularly in crowds (Batty et al., 1999, Helbing, 1992, Helbing and Molnar, 1997), and such models do seem to match various observed human behaviours, such as a tendency to spontaneously form 'lanes' on busy pavements. A more ambitious example is provided by the monumental TRANSIMS (Beckman, 1997, Casti, 1997) where the commuting behaviour of thousands of people (in Albuquerque) is modelled, and virtual 
traffic jams are observed and anticipated. The TRANSIMS model relies on the vast computing resources at the Los Alamos National Laboratories. The model city in this case is populated with agents according to detailed demographic and other data available to the modellers. Thus the model does not attempt to predict how transport patterns are likely to change over time, but shows how the different plans of the current population of commuters, within the existing transport arrangements of a city, are likely to produce congestion and other effects. A more modest model, with a similar split between externally handled, relatively fixed demographic factors, and dynamic behavioural patterns, is the STREETS model of pedestrian shopping behaviour (Schelhorn et al., 1999). Preliminary work linking these kinds of models with Hagerstrand's space-time geography is presented by Huisman and Forer (1998).

\subsection{Economic agent-based models}

In economics, bounded rationality is particularly suitable for investigation by agent-based simulation. Conlisk (1996) provides an extended overview of this terrain, which recognises that the wholly rational utility maximiser of classical economics is a fiction. Rather, people use rules of thumb and various inductive reasoning strategies to decide how to proceed in any particular situation. The classic recent example is provided by Arthur (1994), who describes an agent-based model to investigate a 'toy' problem in this area. A popular bar (which gives Arthur's example its name viz. the 'El Farol problem') is frequented by some proportion of a population of 100 agents. However, there is limited space in the bar, so that a good time is to be had only if no more than 60 people turn up. The model focuses on the inductive reasoning mechanisms by which the population determine whether or not to visit the bar. Each agent uses a set of rules to decide whether to show up or not. These rules are framed as attempts to predict how many other agents will go to the bar. If an agent expects more than 60 other people to go then it won't go, and if it expects 60 or fewer to go, it will. Each agent initially has a set of possible functions relating recent weeks' attendances to an expectation for this week's attendance. An example might be 'the same number of people will go this week as went last week'. As the model runs, agents modify the extent to which they rely on their various rules, according to their accuracy in previous weeks, so that eventually they tend to favour accurate rules. The outcome from this rather "clunky" (Arthur's own term) model of inductive reasoning behaviour, is that attendance at the bar fluctuates unpredictably around the optimal level of 60. Arthur believes this outcome would be robust even with a more sophisticated behavioural model. Arthur has subsequently been involved in work to develop similar psychological models of the stock market (Arthur, 1995, Palmer et al., 1994).

Other economic work using agent-based models includes Kirman (1993), Tesfatsion (1997), Youssefmir and Huberman (1997), and Vriend (1999). It is noteworthy that many of these examine 'path-dependencies' where the historical and spatial development of an economic process may have an effect on the eventual outcome. Related work explores the development of regional specialisation ${ }^{1}$ (Krugman, 1991). These efforts are closely related to a view of the 'economy as an evolving complex system' promoted by the Santa Fe

\footnotetext{
${ }^{1}$ Whilst some might welcome the (belated) arrival of space and time in economic theory, Martin (1999) makes some pertinent points about how this project is being pursued with little reference to the earlier insights of economic geography.
} 
Institute (Anderson et al., 1987, Arthur et al., 1997) through joint meetings between economists, physicists, biologists and mathematicians. That these developments are aspects of a widespread disillusion with neoclassical equilibrium economics is apparent (Arrow, 1994, Kirman, 1989), although other forms of 'evolutionary' economics are less fixated by the 'scientific' method, and instead invoke more interpretative, historical explanations (see David, 1985, for the clearest example, where the 'lock-in' of the QWERTY typewriter keyboard is investigated; see also Argyrous and Sethi, 1996, and Hodgson, 1992 for discussions of the evolutionary trend in economics). The complementary nature of the approaches is clear in earlier work by Arthur (1989) where a less explicitly agent-based approach is used to understand the 'lock-in' described by David. In fact, there is a widespread assumption in economics that the formal mathematical modelling which has characterised much of the 'theory' in the discipline during the postwar period, is destined to be supplanted by modelling and simulation. This view has been (perhaps a little regretfully) endorsed by one of the leading lights of mainstream economic theory (see Hahn, 1991).

\subsection{Sociological agent-based models}

Of the examples we consider, the most exhaustively presented is Epstein and Axtell's (1996) Sugarscape. This model is distinguished from other work in this review in that it not presented as an enquiry into some particular theoretical or policy issue, but as a whole new approach to social science. The authors describe an increasingly elaborate 'artificial world' in which increasingly complex agents have their being. The Sugarscape is a $50 \mathrm{x}$ 50 grid of cells each with some predetermined capacity of 'sugar' which is initially the only resource in the world. Starting with simple gathering behaviour, whereby agents move around the Sugarscape collecting sugar and accumulating 'wealth', increasingly complex behaviours are modelled including trade, war, tribal or cultural traits, inheritance, and disease transmission. Agent behaviour is complicated by each of these extensions of the model. This work is enthusiastic about the prospects of a 'generative social science' in which understanding of some phenomenon is to be judged by the success with which it can be generated from relatively simple individual rules, in an artificial world. The similarity of such models to Schelling's (1969) model of racial segregation is obvious, although it is debatable whether the insistent addition of complicating factors adds anything to our understanding.

Similarly optimistic, yet more thoughtful prospectuses are found in the introductory material in Gilbert and Doran's, (1994) Simulating Societies and Gilbert and Conte's (1995) Artificial Societies. Consideration is also given to some of the wider issues in these books, such as the relationships between structure and agency, and between society and the individual as they are represented in the social simulation community. We return to these matters in section 5 below. For now we note the breadth of the contributions here, ranging from simulation of a fishermen's ( $\underline{\mathrm{sic}}$ ) society (Bousquet et al., 1994) to the emergence of geo-political actors (Axelrod, 1995b). Other sociological applications of the agent modelling approach are found in Axelrod (1995a) who discusses a model of the transmission and maintenance of cultural traits, Gilbert (1997) who presents a model of academic science in which papers are the agents, and in Edmonds (1999) who extends Arthur's El Farol model by including agent-agent social interaction in the decision process. Agent-based models can also be identified in the archaeology and anthropology literature. Simulations of various hunter-gatherer societies have been produced (Doran et al., 1994, 
Lake and Mithen, 1998, Mithen, 1994). Two features of such models can be distinguished. First, at least in part, they are subject to empirical validation by comparison with the archaeological record. Thus, the model can be used to predict where artefacts from a culture are likely to be found, and the archaeological record serves as a check on the theories which underpin the model. A second feature of interest is the attempt in the latter two of these models to explicitly model society and its effects on individuals.

\section{Agent-based models and individualism}

Before examining some of the implications of agent-based models in more detail, it is worth pausing to consider some of the common features of many of the models above. Several characteristics which feature in many models, are interwoven in the discussion in the remainder of this paper. First, agents almost invariably represent individuals. We argue in more detail below that this means that agent-based models often tend strongly towards methodological individualism, and that they need to be understood in this light. We argue, not that methodological individualism is unavoidable, but that it is a frequently unacknowledged assumption of models of this kind. Second, this commitment to individualism is accompanied by a one-way notion of emergence: the social can emerge from the individual but not the other way round. Third, and implicit in both of the previous points, there is often little consideration of the theoretical underpinnings of the model presented. Fourth, and similarly symptomatic of a lack of critical examination of model assumptions, there is a strong commitment to minimal behavioural complexity. This is often presented as a necessary evil. Simplicity is required to make building the model and understanding it possible. Space prevents us from discussing these latter three points in detail, but we return to them in section 5, where we discuss the implications of our critique for agent-based modelling.

In light of the preceding overview of the field, we contend that agent-based models lean very much towards an individualist view of society. The clearest sense of the ontology of many social scientific agent-based models is obtained from Epstein and Axtell's (1996) Sugarscape model already discussed. For them, agent-based models "involve three basic ingredients: agents, and environment or space, and rules." (page 4). Further, "[a]gents are the "people' of artificial societies." (page 5). The environment "is a medium separate from the agents, on which the agents operate and with which they interact." (page 5, original emphasis). Rules govern the way in which agents behave, as well as the way the environment may change over time. There is no social realm in this conception. Rather "fundamental social structures and group behaviours emerge from the interaction of individual agents operating on artificial environments under rules that place only limited bounds on each agent's information and computational capacity." (page 6, original emphasis). This approach is individualist, in that there are only individual rule-bound agents, the aggregate behaviour of whom constitutes society.

The implications of such a reductive ontology are clearest when 'culture' and 'tribes' are added to the model (pages 71-82). Culture is injected in the form of sequences of zeros and ones, with each digit representing some (unspecified) cultural trait. Each agent starts life with one of these 'tags' inherited from its parents. As agents move around the environment they may 'flip' single randomly selected digits in nearby agents tags if they are different from the corresponding digit in their own tag. In this way groups of agents with similar tags 'emerge' and are labelled 'tribes'. This is a completely unsatisfactory 
model of cultural transmission and tribe formation. The root of the problem does not lie in the simplification of reality, but in the failure to allow for the pre-existence of culture and tribes. This is not to say that culture exists independently from individuals, but that culture does pre-exist any individual human. We are born into socio-cultural structures which shape us as individuals. This example also illustrates the one-way notion of emergence which is often found in agent-based models, and which is most readily understood as an acceptance of the truism that society is nothing more than the aggregate activity of individuals. That society is also the resultant of all the previous actions of individuals (living and dead) and that it shapes and constrains our actions and development as individuals is ignored. To make this criticism is not to adopt a naïve collectivism or structural determinism. It is merely to recognise that social structures affect human freedom to act in any particular situation. The effects of society may also be felt in the reflexive ways in which individuals decide to act in response to their perceptions of the social structures in which they are embedded. Thus, society not only constrains human actions, it may also give rise to conscious efforts to change society itself (of course, the social sciences are deeply implicated in such efforts). Ultimately, this model of society is impoverished by its failure to include social structural influences on behaviour, either directly, or by representing the responses of individuals to their perceptions of their social situation.

Of course, the Sugarscape is something of a straw man. Arguably, it is presented in a simplistic way to introduce the concept of agent models to a wide audience. Equally, it is not intended as a detailed model of any real world situation. Nevertheless, many more substantial models fall into the trap of adopting a similarly one-sided individualist view of society and social behaviour. Perhaps, the most significant cases where this happens because they may appear to avoid the individualist charge - involve the introduction of agents' subjective views of their social world. In an interesting discussion of these problems, Gilbert (1995), admits that "[i]t is the case that most, if not all, current simulations of human societies [...] adopt [either holist or individualist] positions, often without making this explicit" (page 146). Gilbert suggests that Giddens's structuration theory provides some ideas as to how agent-based models might be extended to represent social systems more effectively. The key to improving models, Gilbert suggests, is for agents to have some sort of (changing) representation of their social world. Agents could then use this representation in making decisions about their activities. This focuses on Giddens's insistence that "individuals are knowledgeable agents" (Thompson, 1989, page 58). Whether such an approach is sufficient to capture the subtleties of structuration theory is debatable. As presented it sounds more like a model of Hayek's subjectivist approach, wherein "the facts of the social sciences are merely opinions, views held by the people whose actions we study" (von Hayek, 1955, page 28). As Lawson (1997, pages 134-151) argues, this is essentially an individualist model. The problem remains that agents are still the only actually existing entities in the world so modelled. Social structure becomes merely what agents think it is. This does open up new areas for exploration, in particular the implications of agents with different world views interacting with one another. However, it does not allow that social structures really are out there, independent of any view of them held by an agent, or indeed of any view being held of them by any agent. To represent Giddens's theories adequately, without effectively representing social rules and institutions external to agents seems an impossible task. Although rules might be bundled up inside agents from a programming perspective, they would be partly of the form "if you occupy some social position or role then you do $\underline{X}$ ", or "these choices are 
differentially open to you", which effectively turns rules into a representation of social structure, and its real effects. A similar criticism of Giddens's concept of social structure as rules has been made (Thompson, 1989).

This is where the agent-based model approach takes us beyond purely 'technical' considerations and aspects of social theory are implicated. An interesting recent exchange pertinent to the argument is Margaret Archer's $(1988,1995)$ morphogenetic social theory and recent criticism of this work by Anthony King (1999). Archer argues that much previous social theory is flawed because it is guilty of one of 'upwards conflation' (individualism), 'downwards conflation' (collectivism), or 'central conflation' (structuration theory). Respectively, these errors deprive society of any existence independent of 'these people here present', deprive individuals of any autonomy from social structure, or deny the possibility of any interaction between structure and agency (by collapsing them into different aspects of social practices). In these terms, we are here arguing against a form of 'upwards conflation' involved in much agent-based model building. Arguing against Archer, for the reduction of all social structure to individuals, King notes that the central point of "Archer's entire project is the maintenance of a stratified social ontology" (King, 1999, page 205), whereby individuals and social structures both have existence and causal force. Since we are also arguing that attention must be paid to a distinct social realm, King's argument is highly relevant. $\mathrm{He}$ convincingly demonstrates that all those manifestations of social structure which Archer presents as evidence of the prior, independent existence of society can be shown to arise from individuals. Thus, quantitative social phenomena (such as the literacy rate) are only 'other people' (King, 1999, pages 208-211). Similarly, relational emergent structural effects, such as increases in efficiency due to the division of labour, while not reducible to any one individual, clearly are reducible to all the individuals present in any particular context (King, 1999, pages 211-214). Finally, King dismisses Archer's argument that social roles provide definitive evidence of the prior and autonomous existence of society, since "roles can be fully understood in terms of individuals as long as we take a wide enough perspective so as to include all those individuals, in other times and places, who create a role" (King, 1999, page 216, emphasis added).

Having demolished Archer's arguments ${ }^{2}$ (and apparently weakened ours too), King's conclusions are important and worth quoting at some length. First

"sociological questions must always address specifically empirical questions of how individuals constrain one another and not metaphysical ones of how structure constrains or facilitates individual action."

(King, 1999, page 223)

This argues against the existence of an independent social realm. However,,

"practically, a [...] concept of structure can be usefully maintained. In any sociological study, the sociologist will have to situate the specific practices under examination within a wider social and historical context. Theoretically, this

\footnotetext{
2 King goes on to make an interesting point to the effect that Archer's arguments are weakened because she adopts a strong definition of the individual, based on a notion of the pre-existent self independent of society. This point is also relevant and interesting in the context of agent-based models, but takes us well beyond the scope of the current article.
} 
context could come under micro-reduction and we could demonstrate how each institution and economic form is only the complex [outcome] of individual interactions and exchanges over time. Such a reduction could go on ad absurdum, so that the sociologist never actually analysed the practice under consideration."

(King, 1999, page 223)

Thus, crucially

"[A]t some point then, $[\ldots]$ the sociologist is going to have to assume certain background conditions which are not reduced to their micro dimensions. This background might usefully be called 'structure' $[\ldots]$ "

(King, 1999, page 223, emphasis added)

We would argue that similar considerations must be brought to bear in the design and construction of agent-based models. Because models are closed representations of an open world, the spatio-temporal boundaries of any model require the explicit inclusion and modelling of social structures, particularly institutions. and the social roles within them. This requirement can be argued either on the purely practical basis of the need to avoid infinite regress, or on the basis of a firm theoretical commitment to the existence of a distinct social level of existence. King argues for the former; Archer and others (for example Sayer, 1992) would argue for the latter. In either case, we would argue, social structure must be admitted into models explicitly, or explicitly rejected, and the reasons for that rejection explained. Such rejection would either imply that the spatio-temporal boundaries of the model mean that social structure is irrelevant in the context, or that the modeller rejects the notion that the relevant social structures have an existence autonomous from the particular population of agents represented in the model.

\section{Origins of individualism in agent-based models}

Before discussing possible approaches to agent-based modelling which tackle the issues raised above (see section 5), it is worth examining the roots of the individualist bias in agent-based models, as it can be argued that these help to explain why the bias has gone largely unnoticed. In this section we sketch a tentative explanation of the individualist bias in agent-based models. This explanation is two-fold. First, we examine the history of multi-agent systems in the computer sciences and especially in the field of artificial intelligence; second, we point to the current intellectual climate in the social sciences, which favours an individualistic view of society.

\subsection{Computer science / artificial intelligence}

Artificial Intelligence (AI) first appeared as a significant disciplinary division during the 1950s. The term itself was coined at the Dartmouth conference, in 1956 (Huhns and Singh, 1998). From the outset, AI sought to make 'machines that think'. When AI researchers wanted models of agency and social interaction, they looked at then current (largely positivist, individualist, English language) research in the social sciences, and especially at those models which could be easily formalised and represented in computers. This first period saw the development of specialised computer languages (such as LISP and Prolog) and of 'General Problem Solver' programs. However, AI experienced various 
crises in the late 1960s, caused by an attempt to find universal rules to simulate conscious thinking with computers.

As AI emerged from this troubled period, it moved into a period of specialisation and critical self-examination which was marked by the creation of various sub-disciplines, including Distributed AI (DAI). DAI is concerned with agents as computational entities which can interact with each other to solve various kinds of distributed problems. In a review of the state of DAI in the late 1980s, Castillo Hern (1988) mentions the emergence of agents as a major object of investigation in this research. Like AI, DAI was exploring 'problem solving' mechanisms. In DAI, the focus was on co-operation between different components that interact with one another through communication networks. Hence, one of the alternative terms for them was 'node processors'.

According to Huhns and Singh (1998), AI adopted concepts from the psychological and philosophical concepts of agency. These concepts were kicking around in AI from the earlier attempts to create 'intelligent machines'. During the 1970s and the 1980s, initial optimism about building intelligent machines dwindled and was replaced by more narrowly defined problems and possible solutions. During the late 1970s ideas about agency again came to prominence, this time within DAI. While AI drew heavily on psychology, DAI borrowed insights from sociology, organisation theory, economics, philosophies of language, and linguistics. The connection between psychology and philosophy and early AI research is well documented. For example, McCarthy (1988) emphasises the fact that efforts were focused on using the computer as a tool, and that the main use of human behaviour research was in providing hints for the development of computer programs. Although some of the pioneers of AI (such as Simon and Newell) regarded it as a combination of psychology and computer science, McCarthy argues for the view that AI grew primarily as a branch of computer science. It is arguable that McCarthy's position - that AI is merely a computer science sub-discipline - cannot be sustained. If some workers in the field viewed AI as an interdisciplinary research field, and borrowed models from other disciplines, then those engaged in the field would be influenced (knowingly or not) by the ontologies and methodologies of those other disciplines.

With advances in computing technology, particularly the move towards a networked computing environment, and the development of Object Oriented Programming (OOP) techniques in the late 1980s and into the 1990s, the DAI vision became feasible. Agents and multi-agent systems started to mushroom, and soon after broke out of the disciplinary bounds of DAI, to be adopted by many other branches of computer science. The end result of this development history is that when DAI methods proliferated and were adopted across the computer sciences, at least for some social scientists, it appeared to be a neutral 'objective' abstraction well suited to their modelling needs. The implication of this history of the discipline is that early intellectual borrowings from (particularly) post-war English language sociology may have had a significant impact on the approach.

\subsection{Social science and theory}

In the social sciences, some of the more influential analyses of social action as emergent from individual behaviour started to appear in the 1930s and 1940s (see for example Kardiner and Linton, 1940; the recent reprinting of this book is intriguing in itself). This period was also marked by the extensive use of deterministic models in the social sciences. The development of quantitative models (during the so-called 'quantitative revolution') 
continued through to the 1950s and '60s. However, in the late 1960s and into the 1970s many in the social sciences lost interest in these models, instead turning to marxist and other more structuralist approaches. More individualist models and modes of thought (including postmodernism) were rediscovered during the 1980s and 1990s. The views of current prominent theorists in sociology lend both theoretical and empirical support to analyses of society as a collection of interacting agents. For Castells (1996, 1997, 1998), as we move towards an increasingly knowledge and information-based society, the importance of previous social infrastructure is declining. His analysis can be taken to imply that an individualistic view of society is the most likely to be useful. Castells is not alone in this sort of analysis. In a recent series of lectures titled Runaway world, Anthony Giddens (1999) has expressed similar views. Another explanation for the perceived disintegration of society and a move towards more individualistic views, can be related to global political changes and their influence on Western society. Robert Cooper (1999) suggests that the turn towards an individual oriented society is the result of a long and lasting peace in the West. This view echoes Inglehart's (1977) analysis of the origins of the environmental revolution. Inglehart's model connects contextual changes (such as economic prosperity, peaceful existence, increased levels of education and the development of the mass media) to changes in values and capabilities of the individual which in turn, cause changes in national political agendas towards an emphasis on the quality of life and away from class struggle. These analyses focus on the individual agent as a force for change in the social arena. Within geography, we might point to David Harvey's (1989) 'space-time compression' as an explanation for increasingly individualistic perspectives.

The approaches presented are representative of a range of views on the increased fragmentation and individualism of social life. Whichever view is most compelling, there does appear to be general agreement over a need to account for the increasing importance accorded individual preference, opinion, and choice. In the current context, the important point is that the intellectual current is flowing strongly in favour of individualist models.

We have seen, in this section, that it is possible to relate the proliferation in the use of agent-based models to two sources: advances in computer technologies which enable the creation of such models, and an intellectual climate ready to sustain individual-based models and open to claims that we should expect the emergence of social structures from such models. The inter-influence between an individualist view of society as (merely) the aggregate behaviour of large groups of individuals, and software agents and DAI - and through these to agent-based modelling, is clear in the collection The Ecology of Computation (Huberman, 1988). Here, the ideas of Dawkins, Hayek and the ecological and evolutionary views of markets are connected and formalised so that they can be adequately modelled using computers.

\section{Implications for building agent-based models}

We believe that we have demonstrated that agent-based models do not come unburdened by theoretical baggage. This baggage influences the use of agent-based models and should be carefully unpacked when using, implementing, and evaluating such models. Our own interest in this critique was sparked by our experience of the agent-based modelling process, and our intention has been to subject aspects of that process to critical examination. We have focused particularly on the tendency of agent-based models towards an individualistic view of the social world, which often denies social structure independent 
existence or causal impact. Although it would be inaccurate to describe all agent-based models as inherently individualistic, our critique demonstrates that an individualist bias is a prominent issue in agent-based models. Agent-based models are biased towards individualist strands of social theory and thought. Do the traces of these theories blight agent-based models to such an extent that in using them researchers can only uncover the underlying individualist models of society? We hope it is clear that we do not think so. However, it is important for modellers to be aware of the pitfalls, and for sustained efforts to be made to address the issue. In this section we suggest some possible approaches to the building of agent-based models which face up to this problem. We also comment more generally on relevant issues in agent-based model building.

We have argued that whether for practical reasons, or because of a theoretical commitment to a stratified social ontology, it is important that social structure be explicitly represented in agent-based models, or that its omission be justified. It is clear that the requirement for the inclusion of social structure in some explicit sense is crucially dependent on the spatial and temporal boundaries and scales of the model. Thus in our own STREETS model of second-by-second pedestrian behaviour in a town centre, a strong case can be made that many of the social institutions (retailers, urban planners and local government) which impinge on the model are not subject to modification by its evolution, and can be safely ignored. Other social institutions (family and acquaintanceship groups, the police and other authority figures) may be less easily excluded. Certainly, how the model copes with these aspects of the social context deserves scrutiny and explanation (for the record, neither aspect is included in our model, largely because we have not reached the stage of considering these aspects).

Arguably, STREETS models an aspect of human social behaviour which does not in itself much affect more enduring social phenomena such as markets, firms, institutions, the state, and the legal system. Many agent-based models attempt to represent aspects of precisely such phenomena, and it is here where we believe it becomes important to explicitly acknowledge them. Various approaches in the literature are worthy of comment, and deserve wider examination, and further development.

We have already cited the MAGICAL model (Lake and Mithen, 1998) of the behaviour of groups of hunter-gatherers in prehistoric Scotland. Here an explicitly social level of behaviour is introduced, whereby collective decisions to move camp are made. This involves introducing an explicit concept of the tribal group into the infrastructure of the model, and allowing group decisions to affect individual behaviour, although "group activities can only be initiated by individual agents and not by groups themselves." (page 260) The mechanics of this process are a little awkward, and certainly the infrastructure of the approach is rather inflexible. This reflects the focus of the model on spatial outcomes and not on the interactions between individual and group behaviours. Nevertheless, we believe such explicit acknowledgement of the social realm represents progress.

Another ongoing approach to this issue is to be found in attempts to model organisations. Prietula et al. (1998) provide a useful overview of work in this field. A particularly interesting approach is suggested by Fox et al. (1998) who introduce an organisational object taxonomy which includes organisations, organisation-goals, divisions, roles, authority and so on. Agents can play roles, which potentially give them authority over other agents playing other roles. Agents may play one or more roles, and roles may be 
associated with many goals. The authors correctly identify empowerment and authority as critical aspects of this approach, since these identify which roles (and hence which agents) are enabled to perform various actions. A particularly important aspect of more developed models of this kind would be the issue of whether and which roles are empowered to define new roles and authorities. This would become significant if there was an interest in examining processes of change of institutional and social structure.

Finally, an attempt to face the issue of institutions and organisations which act to sustain and maintain themselves through the activities of their constituent agents, is to be found in the work of Cristiano Castelfranchi and his colleagues (Castelfranchi, 1997, 1998, Conte and Castelfranchi, 1995). They propose a framework in which social actions are distinct from simple actions, and in which delegation, goal adoption, power and authority are significant. This framework may enable less formal institutions and structures (for example the family) to be modelled, something which would be difficult using the organisational taxonomy approach. Although this work is still at the conceptual stage in the (D)AI community, so that it is likely to be some time before the ideas are widely implemented, we believe that this literature deserves wider attention from the agent-based modelling community.

Interestingly Castelfranchi is sceptical about prevalent notions of emergence in the agentbased modelling community:

“... emergence simply relative to an observer [...] or a merely accidental cooperation (like stars 'cooperate' [in] the emergence of our beautiful constellations) are not enough. We need an emerging structure playing some causal role in the system [...]; not merely an epiphenomenon. [...] Possibly we need even more than this: really self-organizing emergent structures. Emergent organisations [which] reproduce, maintain, stabilize themselves [...]"

(Castelfranchi 1997, page 1575, original emphasis).

We share this scepticism. In fact, much of the importance of the concept of emergence seems to lie in its rhetorical power. Modellers can point to emergent aggregate phenomena in purely individual models and claim 'we didn't put that in the rules... it emerged'. This sort of claim can be quite convincing. However, even proponents of the concept note that "the mapping from micro-rules to macrostructure could be many to one" (Epstein and Axtell, 1996, page 20), so that the fact of the emergence of plausible aggregate structures proves very little. Furthermore, the claim of emergence can serve to hide the process of searching for the set of rules which produced the aggregate structures: presumably in the course of model construction rules were tried which failed to produce the required structures. In this sense the aggregate behaviours have not emerged: they have been designed. Another useful antidote to this approach is provided by Gilbert (1995), who suggests that the much-feted "emergence may be neither a stable nor an especially interesting property of [agent-based models]: what are interesting are the systems' macro properties and the relationship of those macro properties to the micro ones." (page 150). This points to the possibility that social structures (the macro) can influence individual behaviour (the micro) as well as the other way round, a concept in keeping with structuration theory (Gilbert's own concern) and other non-individualist social theory. Work such as that of Cohen et al. (1998) is beginning to explore how some structural aspects of the relations between agents can affect the sorts of agent behaviours which are observed, although it is noticeable that the title of this paper still endorses one-way (only) 
emergence of the social from the individual. In itself, this seems to indicate that a shift in emphasis, such as Gilbert suggests, could help to change the questions which researchers in the field are asking, and hence the whole emphasis of the approach. Both Gilbert's and Castelfranchi's remarks, to the effect that research should focus on two-way relationships between macro and micro structures, are encouraging.

Moving on to another aspect of the agent-based approach, we have referred to the lack of critical examination of many model assumptions, and also to a widespread commitment to minimal behavioural complexity in agent behaviour. This reflects a general need for model assumptions to be described explicitly. Agent-based models in social science are usually built as closed software systems. While writing program code for these systems, programmers unavoidably include all kinds of assumptions which are never made explicit. Regardless of the rigour of system documentation, assumptions are hidden in the model code. An interesting insight into this problem is provided by multi-agent systems, and the problem of communication between different agents. In this case, unlike the closed worlds of models, there is a requirement for communication between agents which may have been developed separately. For this purpose, a special communication language has been developed, known as Knowledge Query and Manipulation Language (KQML) (Genesereth and Ketchpel, 1994). One of the more interesting elements of messages in KQML is the integration of 'ontology' as part of the message. Ontology, for this purpose is defined as "specification schemes for describing concepts and their relationships in a domain of discourse" (Finnin et al., 1997). These ontologies are usually described in a scheme based on first-order logic. The importance of this to agent-based modelling should be clear, but in many current agent-based models any underlying ontology is hidden in the code and not discussed in detail in the presentation of model findings.

This is likely to become a more serious problem as various agent-based tools become more widespread. For example, the introduction to Swarm states that it aims to provide an objective experimental environment:

"Swarm is an efficient, reliable, reusable software apparatus for experimentation. If

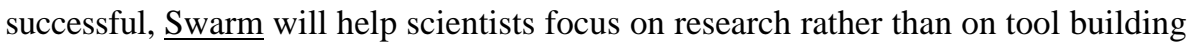
by giving them a standardized suite of software tools that provide a well equipped software laboratory."

(Minar et al., 1996)

Even in its current state as an open-ended open-source product this approach means that many of the assumptions underlying the Swarm system may become invisible to modellers (Curry, 1998 has identified a related problem with the black boxes of GIS). Attempts to build meta-languages which simplify the modelling process (such as MAML Gulyás and Kozsik, 1999) and require less 'real programming' may exacerbate the problem. Rigid 'black-box' environments like StarLogo - in which only two kinds of entity, turtles and patches (agents and a spatial grid), exist - are even more problematic, when used for serious model building. Again, these tools and developments are not bad in themselves. However they are prone to use in non-reflective and uncritical ways, which is precisely the criticism we are making of agent-based models in general. Again, the only response to this problem is to insist on the need for modellers to be more explicit about the assumptions they bring to building their models. 
A further implication of the reductive ontology of agent-based models is a lack of spatial concepts in basic theories about agents. While many of the models we discuss were developed within the aspatial discipline of economics, using agent-based models in research areas which examine spatial phenomena and relations in space explicitly is more problematic. Treuil (1998) addresses this problem directly, without hiding the complexity of this task. This seems to be an area in which geographers are well placed to make a contribution.

\section{Conclusions}

Agent-based models are powerful tools, with potentially widespread application in geographic research, policy development, and decision support. It is already apparent that such models are likely to become a significant weapon in the armoury of strategic planners in both the state and corporate sectors, so critical engagement with them is, we feel, timely.

Researchers who use these systems should be aware of the way that they are constructed, and what is (and is not) achievable with them. In particular, it may be that more effort should be devoted to compensating for a noticeable bias in the literature towards individualistic models by introducing new computational structures to represent theories about social practices and structures. The individualist approach often adopted in agentbased models is highly contestable. Its dominance of agent-based models in economics is unsurprising given the stance of that discipline over many years (intriguingly, it is a physicist who expresses the most disquiet about the limits of the approach in the early Santa Fe contributions: see Anderson, 1987). It is more disappointing to find an easy acceptance of individualism in more sociologically motivated models.

It has been stressed that in spite of superficial similarities between agent-based modelling ontology and current social theory, there are significant differences. For Castells and Giddens (and, emphatically, Harvey) the fact that the world can be understood in terms of individuals interacting through various networks does not mean that other aspects of social structure - family, community, institutions, capital, the state - are no longer of any importance. These social structures still play major roles in society. We conclude that if agent-based models are to have greater value as tools of social scientific enquiry, then they must be informed by contemporary social theory (whether structurationist, realist or morphogenetic), which recognise the dual nature of individuals and societies, and their mutually constitutive roles. We have indicated a number of current research directions which are beginning to face these problems. By identifying these issues, and presenting them to a wider audience, we hope we can contribute to the interdisciplinary collaboration which is undoubtedly required, if this interesting new approach is to fulfil its potential. 


\section{References}

Anderson, P W, 1987, "A physicist looks at economics: an overview of the workshop", in The Economy as an Evolving Complex System Eds P W Anderson, K J Arrow, D Pines (Addison-Wesley, Redwood City CA)

Anderson, P W, Arrow, K J, Pines, D Eds, 1987 The Economy as an Evolving Complex System (Addison-Wesley, Redwood City CA)

Archer, M, 1988 Culture and Agency: the place of culture in social theory (Cambridge University Press, Cambridge)

Archer, M S, 1995 Realist Social Theory: the morphogenetic approach (Cambridge University Press, Cambridge, England)

Argyrous, G, Sethi, R, 1996, "The theory of evolution and the evolution of theory: Veblen's methodology in contemporary perspective" Cambridge Journal of Economics 20(4) 475-495

Arrow, K J, 1994, "Methodological individualism and social knowledge" American Economic Association Papers and Proceedings 84(2) 1-9

Arthur, W B, 1989, "Competing technologies, increasing returns, and lock-in by historical events" Economic Journal 99(394) 116-131

Arthur, W B, 1994, "Inductive reasoning and bounded rationality" American Economic Association Papers and Proceedings 84 406-411, online at http://www.santafe.edu/arthur/Papers/El_Farol.html

Arthur, W B, 1995, "Complexity in economic and financial markets" Complexity 1(1) 2025

Arthur, W B, Durlauf, S, Lane, D A Eds, 1997 The Economy as an Evolving Complex System II (Addison-Wesley, Reading MA)

Axelrod, R, 1995a, "The convergence and stability of cultures: local convergence and global polarization", Institute of Public Policy Studies Discussion Paper 375, University of Michigan

Axelrod, R, 1995b, "A model of the emergence of new political actors", in Artificial Societies Eds N Gilbert, R Conte (UCL Press, London), pp 19-39

Batty, M, Jiang, B, Thurstain-Goodwin, M, 1999, "Local Movement: Agent-Based Models of Pedestrian Flows", Working Paper 4, Centre for Advanced Spatial Analysis, University College London

Beckman, R J, 1997, "The TRansportation ANalysis SIMulation System (TRANSIMS) The Dallas-Ft. Worth Case Study", Los Alamos Unclassified Report LAUR-974502LANL, Los Alamos National Laboratory (online at $h t t p: / / w w w-$ transims.tsasa.lanl.gov/)

Bousquet, F, Cambier, C, Mullon, C, Morand, P, Quesniere, J, 1994, "Simulating fishermen's society", in Simulating Societies Eds N Gilbert, J Doran (UCL Press, London), pp 143-162

Castelfranchi, C, 1997, "Modeling social interaction for AI agents", in IJCAI-97. Proceedings of the Fifteenth International Joint Conference on Artificial Intelligence Ed M E Pollack (Morgan Kaufman Publishers, San Francisco, CA), pp 1567-1576 
Castelfranchi, C, 1998, "Modelling social action for AI agents" Artificial Intelligence 103 157-182

Castells, M, 1996 The Rise of the Network Society (Blackwell, Malden, MA)

Castells, M, 1997 The Power of Indentity (Blackwell, Cambridge, MA)

Castells, M, 1998 End of Millennium (Blackwell, Malden, MA)

Casti, J L, 1997 Would-be worlds How simulation is changing the frontiers of science (John Wiley \& Sons Inc., New York)

Castillo Hern, L E, 1988, "On distributed artificial intelligence" Knowledge Engineering Review 3(1) 21-57

Cohen, M D, Axelrod, R, Riolo, R L, 1998, "The emergence of social organization in the Prisoners' Dilemma: how context preservation and other factors promote cooperation", Working Paper 99-01-002, Santa Fe Institute (online at http://www.santafe.edu/sfi/publications/99wplist.html)

Conlisk, J, 1996, "Why bounded rationality?" Journal of Economic Literature 34(2) 669700

Conte, R, Castelfranchi, C, 1995 Cognitive and Social Action (UCL Press, London)

Conte, R, Hegselmann, R, Terna, P Eds, 1997 Simulating Social Phenomena (SpringerVerlag, Berlin)

Cooper, R, 1999, "The long peace" Prospect(40) 22-25

Curry, M R, 1998 Digital places : living with geographic information technologies (Routledge, London ; New York)

David, P A, 1985, "Clio and the economics of QWERTY" American Economic Association Papers and Proceedings 75(2) 332-337

Doran, J, Palmer, M, Gilbert, N, Mellars, P, 1994, "The EOS project: modelling Upper Paleolithic social change", in Simulating societies Eds N Gilbert, J Doran (UCL Press, London), pp 195-221

Edmonds, B, 1999, "Modelling socially intelligent agents" Applied Artificial Intelligence 12 677-699

Epistemology and Learning Group, 1999, "StarLogo", http://el.www.media.mit.edu/people/starlogo/, accessed on 12 January

Epstein, J M, Axtell, R, 1996 Growing Artificial Societies: Social Science from the Bottom Up (Brookings Press \& MIT Press)

Finnin, T, Labrou, Y, Mayfield, J, 1997, "KQML as an agent communication language", in Software Agents Ed J M Bradshaw (AAAI Press, Menlo Park, CA), pp 291316

Fox, M S, Barbuceanu, M, Gruninger, M, Lin, J, 1998, "An organizational ontology for enterprise modeling", in Simulating Organizations: computational models of institutions and groups Eds M J Prietula, K M Carley, L Gasser (AAAI Press / MIT Press, Menlo Park CA / Cambridge MA), pp 131-152

Genesereth, M R, Ketchpel, S P, 1994, "Software Agents" Communications of the ACM 37(7) 48-53

Giddens, A, 1999, "Runaway World", http://news.bbc.co.uk/hi/english/static/events/reith_99/, accessed on 5 July 1999 
Gilbert, N, 1995, "Emergence in social simulations", in Artificial Societies Eds N Gilbert, R Conte (UCL Press, London), pp 144-156

Gilbert, N, 1997, "A simulation of the structure of academic science" Sociological Research Online 2(2), online at http://www.socresonline.org.uk/socresonline/2/2/3.html

Gilbert, N, Conte, R Eds, 1995 Artificial Societies: the computer simulation of social life (UCL Press, London)

Gilbert, N, Doran, J Eds, 1994 Simulating Societies: the computer simulation of social phenomena (UCL Press, London)

Gilbert, N, Troitzsch, K G, 1999 Simulation for the Social Scientist (Open University Press, Buckingham)

Gulyás, L, Kozsik, T, 1999, "Multi-agent modeling language", http://www.syslab.ceu.hu/maml/maml.html, accessed on 12 January

Hahn, F, 1991, "The next hundred years" Economic Journal 101(404) 47-50

Harvey, D, 1989 The Condition of Postmodernity (Blackwell, Cambridge, MA)

Helbing, D, 1992, "A fluid dynamic model for the movement of pedestrians" Complex Systems 6 391-415, online at http://www.theo2.physik.unistuttgart.de/helbing.html

Helbing, D, Molnar, P, 1997, "Self-organization phenomena in pedestrian crowds", in Self-organization of complex structures: From individual to collective dynamics Ed F Schweitzer (Gordon \& Beach, London)

Hodgson, G M, 1992, "The reconstruction of economics: is there still a place for neoclassical theory?" Journal of Economic Issues 36(3) 749-767

Huberman, B A Ed, 1988 The Ecology of Computation (Horth-Holland, Amsterdam)

Huhns, M N, Singh, M P Eds, 1998 Reading in Agents (Morgan Kaufman Publishers, San Francisco, CA)

Huisman, O, Forer, P, 1998, "Computational agents and urban life spaces: a preliminary realisation of the time-geography of student lifestyles", presented at Third International Conference on GeoComputation, University of Bristol, 17-19 September (online at http://www.geog.port.ac.uk/geocomp/geo98/68/gc_68a.htm)

Inglehart, R, 1977 The Silent Revolution : Changing Values and Political Styles Among Western Publics (Princeton University Press, Princeton, NJ)

Kardiner, A, Linton, R, 1940 The individual and his society : the psychodynamics of primitive social organization (Greenwood Press, Westport, Conn.)

King, A, 1999, "Against structure: a critique of morphogenetic social theory" Sociological Review 47(2) 199-227

Kirman, A, 1989, "The intrinsic limits of modern economic theory: the emperor has no clothes" Economic Journal 99 126-139

Kirman, A, 1993, "Ants, rationality, and recruitment" Quarterly Journal of Economics 108(1) $137-156$

Krugman, P, 1991, "Increasing returns and economic geography" Journal of Political Economy 99(3) 483-499 
Lake, M, Mithen, S, 1998, "The MAGICAL (Multi-Agent Geographically Informed Computer AnaLysis) Project: Integrating simulation modelling and GIS analysis in archaeology with an application to Mesolithic Scotland" NERC GR3/9540, Department of Archaeology, University of Reading

Lawson, T, 1997 Economics and Reality (Routledge, London)

Levy, S, 1992 Artificial life : the quest for a new creation (Pantheon Books, New York)

Lomuscio, A, 1999, "Intelligent agents - Introduction" Crossroads 5(4) 2

Martin, R, 1999, "The new 'geographical turn' in economics: some critical reflections" Cambridge Journal of Economics 23(1) 65-91

McCarthy, J, 1988, "Book review on the Question of Artificial Intelligence: Philosophical and Sociological Perspectives" Annals of the History of Computing 10(3) 224-229

Minar, N, Burkhart, R, Langton, C, Askenazi, M, 1996, "The Swarm simulation system: a toolkit for building multi-agent simulations", Working Paper 96-06-042, Santa Fe Institute (online at http://www.swarm.org/pubs.html\#AEN2690)

Mithen, S, 1994, "Simulating prehistoric hunter-gatherer societies", in Simulating societies Eds N Gilbert, J Doran (UCL Press, London), pp 165-193

Palmer, R G, Arthur, W B, Holland, J H, LeBaron, B, Tayler, P, 1994, "Artificial economic life: a simple model of a stockmarket" Physica D 75 264-274

Prietula, M J, Carley, K M, Gasser, L Eds, 1998 Simulating Organizations: computational models of institutions and groups (AAAI Press / MIT Press, Menlo Park CA / Cambridge MA)

Resnick, M, 1994 Turtles, Termites, and Traffic Jams (MIT Press, Cambridge MA)

Reynolds, C W, 1987, "Flocks, Herds, and Schools: A Distributed Behavioral Model" Computer Graphics 21(4) 25ff, online at http://hmt.com/cwr/ps/SIGGRAPH87.ps

Rodrigues, A, Raper, J, 1998, "Defining spatial agents", in Spatial multimedia and virtual reality Eds A S Camara, J Raper (Taylor \& Francis, London), online at http://helios.cnig.pt/ armanda/html/Livro.html

Sayer, A, 1992 Method in Social Science: A realist approach (Routledge, London)

Schelhorn, T, O'Sullivan, D, Haklay, M, Thurstain-Goodwin, M, 1999, "STREETS: an agent-based pedestrian model", presented at Computers in Urban Planning and Urban Management, Venice, 8-11 September (online at http://www.casa.ucl.ac.uk/ david/AnAgent.pdf)

Schelling, T C, 1969, "Models of segregation" American Economic Association Papers and Proceedings 59(2) 488-493

Sichman, J S, Conte, R, Gilbert, N, 1998, "Multi-agent systems and agent-based simulation", presented at Lecture Notes in Artificial Intelligence, Berlin

Tesfatsion, L, 1997, "How economists can get ALife", in The Economy as an Evolving Complex System, II Eds W B Arthur, S Durlauf, D Lane (Addison-Wesley), pp 533-564

Thompson, J B, 1989, "The theory of structuration", in Social Theory of Modern Societies: Anthony Giddens and his critics Ed D Held (Cambridge University Press, Cambridge), pp 56-76 
Treuil, J P, 1998, "Space models and agent-based universe architecture", in Tasks and Methods in Applied Artificial Intelligence Eds A P del Pobil, J Mira, M Ali), pp 795-804

von Hayek, F A, 1955, "Scientism and the study of society", in The counter-revolution of science: studies in the abuse of reason (The Free Press, New York), pp 11-102

Vriend, N J, 1999, "Was Hayek an Ace?", Department of Economics Working Paper 403, Queen Mary and Westfield College, University of London (online at http://www.qmw.ac.uk/ ugte173/)

Westervelt, J D, Hopkins, L D, 1999, "Modeling mobile individuals in dynamic landscapes" International Journal of Geographical Information Science 13(3) 191-208

Youssefmir, M, Huberman, B A, 1997, "Clustered volatility in multiagent dynamics" Journal of Economic Behaviour and Organization 32(1) 101-118 